\title{
Results of CUORE-0 and Prospects of the CUORE Experiment
}

\section{Kyungeun Lim*†}

Yale University

E-mail: kyungeun. limeyale.edu

CUORE-0 is a cryogenic detector that uses an array of tellurium dioxide bolometers to search for neutrinoless double-beta decay of ${ }^{130} \mathrm{Te}$. The detector consists of $52 \mathrm{TeO}_{2}$ crystal bolometers held in a ultra-pure copper frame and it was assembled using the new low-background techniques developed for CUORE. Using bolometers operated at $\sim 10 \mathrm{mK}$ provides excellent energy resolution $(<0.2 \%$ FWHM) at the neutrinoless double-beta decay Q-value. CUORE-0 is located at the Laboratori Nazionali del Gran Sasso in Italy and has been taking data since March 2013. We will present the experiment and its neutrinoless double-beta decay search results with a $9.8 \mathrm{~kg} \cdot \mathrm{yr}$ exposure of ${ }^{130} \mathrm{Te}$. We will also discuss the prospects of CUORE, which has a ${ }^{130} \mathrm{Te}$ mass 19 times greater than that of CUORE-0. CUORE is in the final stages of the construction and scheduled to begin data-taking in 2016 .

38th International Conference on High Energy Physics 3-10 August 2016

Chicago, USA

* Speaker.

${ }^{\dagger}$ On behalf of the CUORE collaboration. 


\section{Introduction}

Neutrinoless double-beta decay $(0 v \beta \beta)$ is a hypothetical rare nuclear decay process which has not been decisively observed yet [1]. $0 v \beta \beta$ searches provide an exclusive probe into the Majorana nature of neutrinos, insights on their their hierarchy and mass, and test lepton number violation. It also supports theories that possibly explain the matter dominating Universe through leptogenesis [2]. Due to its significant impact on our understanding beyond the Standard Model of particle physics and the Universe, world wide effort to search for $0 v \beta \beta$ with various detectors and relevant techniques is actively on-going. CUORE, Cryogenic Underground Observatory for Rare Events, is one of the today's leading $0 v \beta \beta$ experiments. We will discuss the physics prospects of CUORE, as well as the results from its predecessor experiment, CUORE-0, in the following sections.

\section{The CUORE Experiment}

CUORE is a cryogenic detector consisting of $988{ }^{\text {nat }} \mathrm{TeO}_{2}$ bolometers arranged in 19 towers, operated at $\sim 10 \mathrm{mK}$. CUORE is located in the Hall A of the Laboratori Nazionali del Gran Sasso (LNGS) underground facility in Italy, whose average overburden is $\sim 3600$ m.w.e.. The primary physics goal of CUORE is search for neutrinoless double-beta decay $(0 v \beta \beta)$ of ${ }^{130} \mathrm{Te}$. With $5 \mathrm{keV}$ Full Width Half Maximum (FWHM) at the Q-value of $0 v \beta \beta, 0.01$ counts/(keV·kg.yr) at the region of interest (ROI), and 5 years of data accumulation, the projected half-life sensitivity to ${ }^{130} \mathrm{Te} 0 v \beta \beta$ is $9.5 \times 10^{25} \mathrm{yr}(90 \%$ C.L.). This half-life corresponds to effective Majorana neutrino mass of $50-130 \mathrm{meV}$. In addition to being a competitive $0 v \beta \beta$ search, CUORE is also suitable for other rare event searches such as Weakly Interacting Massive Particle (WIMP) dark matter direct detection.

CUORE exploits bolometric technique, which measures energy deposition of a particle interaction in the detector through temperature rise. Bolometric technique provides excellent energy resolution, resulting in the suppression of $2 v \beta \beta$ background contribution to the $0 v \beta \beta$ ROI. We instrument each bolometer with a single neutron transmutation doped (NTD) germanium thermistor and one silicon Joule heater, to read the signal from the crystal and to correct the thermal gain drift due to the temperature variation, respectively. Tower configuration, schematic of a bolometer, and an example pulse of CUORE-0 is shown in Figure 1 of [3].

CUORE subsystems includes cryostat, detector calibration system, external shields, electronics, and others. The details can be found in the publication section of [4].

\section{The CUORE-0 Experiment}

CUORE- 0 is a first tower produced by CUORE assembly procedure and new cleaning technique [5]. CUORE- 0 consists of $52 \mathrm{TeO}_{2}$ crystal bolometers with a total detector mass of $39 \mathrm{~kg}$ $\left({ }^{130} \mathrm{Te}\right.$ mass of $\left.11 \mathrm{~kg}\right)$, held in a ultra-pure copper frame. While CUORE-0 is a stand alone experiment to perform its own rare event searches, CUORE-0 also acted as a technical prototype to test data acquisition (DAQ) and analysis framework for CUORE. Its performance and physics results are explained in the following subsections. Analysis details especially on $0 v \beta \beta$ results can be found in [6]. 


\subsection{Detector Performance}

The projected sensitivity of CUORE to $0 v \beta \beta$ requires that background level at ROI and energy resolution at the Q-value of $0 v \beta \beta\left(\mathrm{Q}_{\beta \beta}\right)$ to be 0.01 counts $/(\mathrm{keV} \cdot \mathrm{kg} \cdot \mathrm{yr})$ and $5 \mathrm{keV}$, respectively. CUORE-0 demonstrated that achieving these detector performance goals are within reach.

While the total background level measured at ROI is $0.058 \pm 0.004$ (stat.) \pm 0.002 (syst.)counts/(keV·kg.yr) [7], 76\% of the contribution is coming from $\gamma$ background [8], originated from the radioisotopes in the outer shields of the cryostat, which also used for Cuoricino, the predecessor experiment. CUORE cryostat is made of more radio-pure materials with ancient roman lead shields near the detector region. In addition, the anti-coincidence requirement for CUORE is more stringent than that of CUORE-0 due to 19 times more crystals and we are confident that we could achieve CUORE background goal.

To evaluate the energy resolution at $\mathrm{Q}_{\beta \beta}$, we use $2615 \mathrm{keV} \gamma$-rays from ${ }^{208} \mathrm{Tl}$. Fig. 1 shows the details on how to obtain the energy resolution taking into account of the pulse shape and expected spectral components. The distribution of the energy resolutions evaluated on the $2615 \mathrm{keV}$ calibration peak for each bolometer and dataset in CUORE-0 can be found in Fig. 25 of [9], which results in effective mean of the FWHM values of $4.9 \mathrm{keV}$.

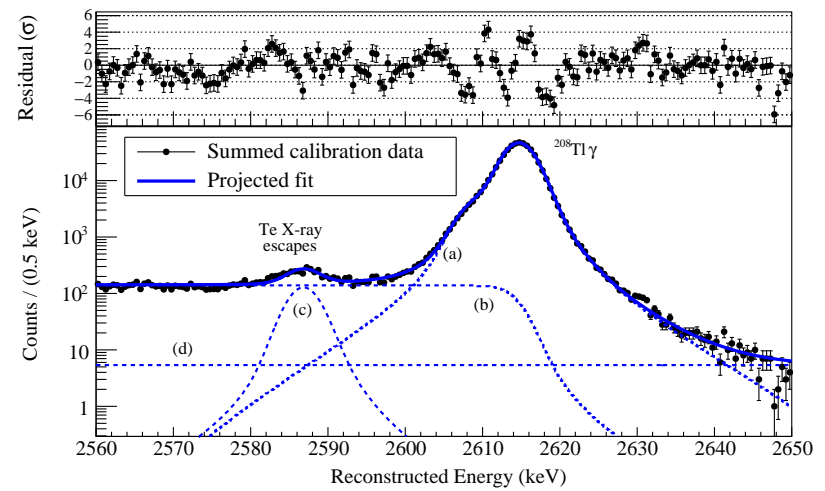

Figure 1: Bottom: Calibration data near the $2615 \mathrm{keV}{ }^{208} \mathrm{Tl} \gamma$-ray line, integrated over all bolometerdatasets. The solid blue line is the projection of the unbinned extended maximum likelihood (UEML) fit, described in [7]. In addition to the double-Gaussian lineshape for each bolometer-dataset, the fit function includes terms to model a multiscatter Compton continuum, a $\sim 30 \mathrm{keV}$ Te X-ray escape peak, and a continuum background; these components, summed over all bolometer-datasets, are indicated by the blue dashed lines (a), (b), (c), and (d), respectively. Top: Normalized residuals of the data and the best-fit model. Figure from [7].

\subsection{Results on the $0 v \beta \beta$ Search}

We have run CUORE-0 till the sensitivity of $T_{1 / 2}^{0 v \beta \beta}$ surpasses the 90\% C.L. limit set by Cuoricino, $2.8 \times 10^{24} \mathrm{yr}[10]$. With the obtained background level at ROI and energy resolution mentioned in Sec. 3.1, we perform the best fit on the unblinded spectrum taking into account of the contribution from the flat background, summed peak of two $\gamma$-rays from ${ }^{60} \mathrm{Co}$, and hypothetical $0 v \beta \beta$ peak at $\mathrm{Q}_{\beta \beta}$ (see Figure 3 of [7]). We obtain Bayesian upper limit at $\Gamma_{0 v}<0.25 \times 10^{-24} \mathrm{yr}^{-1}$, or $2.7 \times 10^{24} \mathrm{yr}$ (statistical uncertainty only) with $90 \%$ C.L., while the median $90 \%$ C.L. lower-limit sensitivity for $T_{1 / 2}^{0 v \beta \beta}$ is $2.9 \times 10^{24} \mathrm{yr}$. 
To report the final results, we combine CUORE-0 results with the Cuoricino results. The profile likelihoods can be found in Figure 4 of [7]. We find no evidence for $0 v \beta \beta$. The combined Bayesian $90 \%$ C.L. limit is $4.0 \times 10^{24} \mathrm{yr}$. We interpret Bayesian limit using the models for $0 v \beta \beta$ mediated by light Majorana exchange, which translate into $\mathrm{m}_{\beta \beta}<270-760 \mathrm{meV}$. Figure 2 shows allowed regions for $\mathrm{m}_{\beta \beta}$ for normal and inverted hierarchies, along with the exclusion limits set by CUORE- 0 and other experiments.

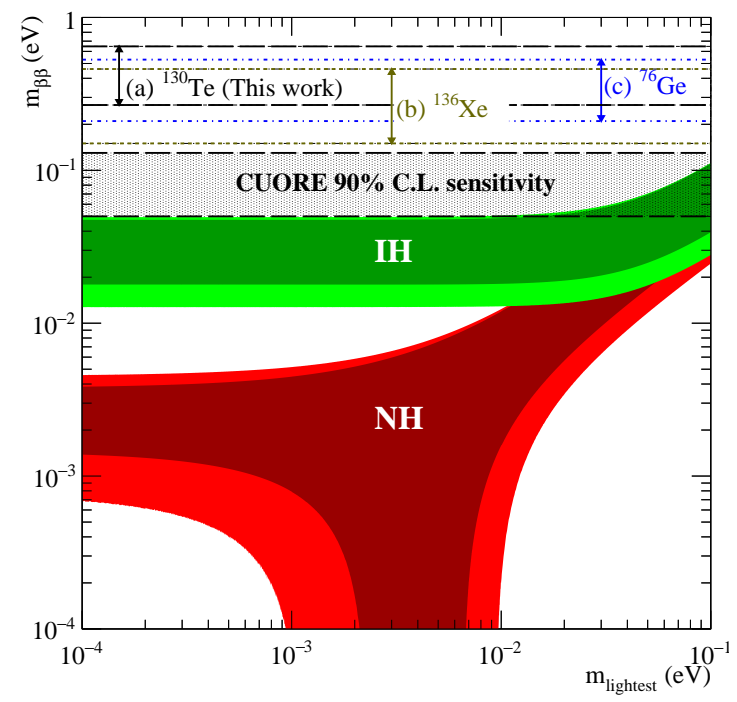

Figure 2: The allowed regions for $m_{\beta \beta}$ for the normal hierarchy (NH) and inverted hierarchy (IH). $90 \%$ confidence upper limits on the effective Majorana mass, $m_{\beta \beta}$, from experiments with ${ }^{76} \mathrm{Ge}[11],{ }^{136} \mathrm{Xe}$ $[12,13]$ and ${ }^{130} \mathrm{Te}[7]$. The width of the upper limit is dominated by the uncertainty in the NME $[14,15,16$, $17,18,19]$. The shaded area is the expected CUORE $90 \%$ sensitivity after 5 years of live time. Figure from [7].

\subsection{Results on the Measurement of the $2 v \beta \beta$ Half-life of ${ }^{130} \mathrm{Te}$}

To construct the background model of CUORE- 0 , we use the results from the radio-assay measurement of detector materials, cosmogenic activation measurements, and CUORE-0 data analysis to infer bulk and surface contamination of $\alpha$ and $\gamma$ backgrounds, as input of the simulation. The GEANT4-based Monte Carlo simulation used implements detailed detector geometry and response, such as energy resolution, threshold of each bolometer, and coincidence window for the multiplicity requirement. Its performance was verified through making comparison of the spectrum with calibration data. Details on how to construct of CUORE-0 background model can be found in [8].

Relying on the CUORE-0 background model, we obtain the $2 v \beta \beta$ half-life of ${ }^{130}$ Te by performing Bayesian fit on the spectrum using JAGS (Just Another Gibbs Sampler). We use variable binning in the histogram to reduce the effects of statistical fluctuation and line shape of the peaks. By identifying all the background sources, along with thorough investigation on the possible sources for the contribution on the systematic uncertainty, $2 v \beta \beta$ half-life of ${ }^{130} \mathrm{Te}, T_{1 / 2}^{2 v \beta \beta}$, is measured to be $8.2 \pm 0.2$ (stat.) \pm 0.6 (syst. $) \times 10^{26} \mathrm{y}$ [8]. This provides the most precise measurement to date on this quantity. Figure 3 shows the obtained $2 v \beta \beta$ contribution in the CUORE- 0 spectrum. 


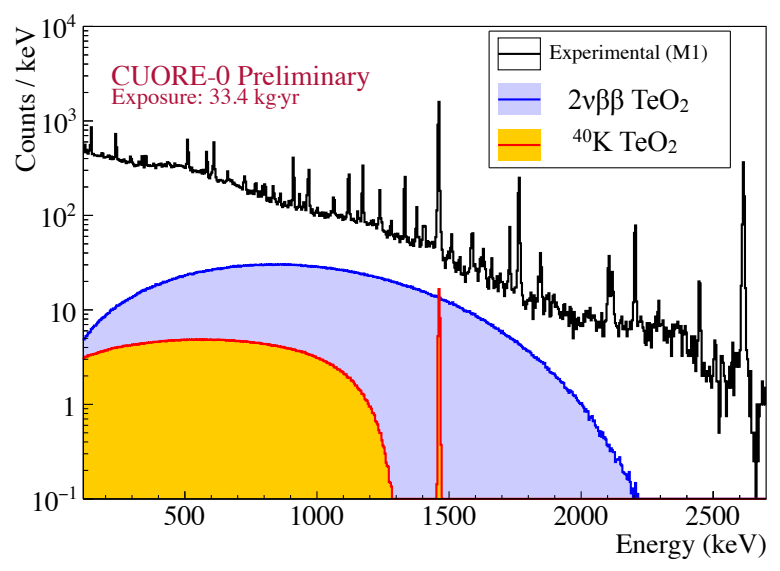

Figure 3: CUORE- $0 \mathscr{M}_{1}$ compared to the $2 v \beta \beta$ contribution predicted by the reference fit and the radioactive source that has the strongest correlation with $2 v \beta \beta,{ }^{40} \mathrm{~K}$ in Crystal bulk. Figure from [8].

\subsection{Prospects on the WIMP Dark Matter Search}

While the primary physics goal of CUORE- 0 is search for $0 v \beta \beta$, successful background mitigation, along with continuous data acquisition make CUORE-0 also suitable for other low-energy, rare event searches such as dark matter. In addition, nuclear quenching factor similar to unity in the bolometer also benefits to reach low energy threshold required in the direct WIMP dark matter detection.

Currently analysis on the search for WIMP-induced annual modulation with CUORE-0 is under finalization, and expected report the results soon. Figure 4 shows the preliminary predictions on the sensitivities of CUORE-0 and CUORE, using the obtained energy threshold for each bolometer-dataset pair.

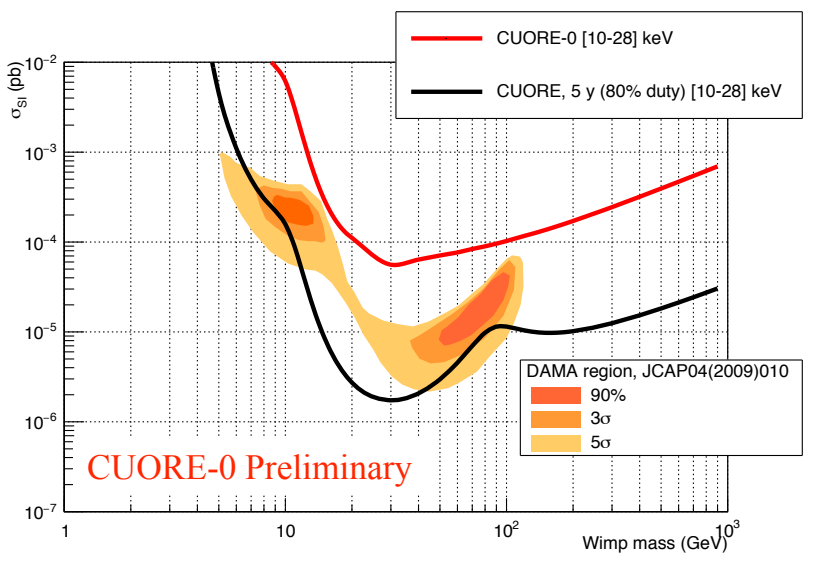

Figure 4: Sensitivity of CUORE-0 (red solid) on the spin-independent elastic WIMP-nucleon cross section as a function of WIMP mass, using the obtained bolometer-dataset dependent energy thresholds. CUORE sensitivity is also shown assuming 5 years of data-taking with $80 \%$ of duty cycle. Contour indicates the WIMP observation region from the interpretation of the DAMA experiment results [20]. 


\section{Outlook}

\subsection{Status of CUORE}

After successful commissioning of the cryostat, we have finished installation of the detector in the cryostat in late August of 2016, as shown in Fig. 5. At the time of writing, we are closing the vessels and plan to start to cool down the detector in mid November, aiming to begin operation at the base temperature in mid December.

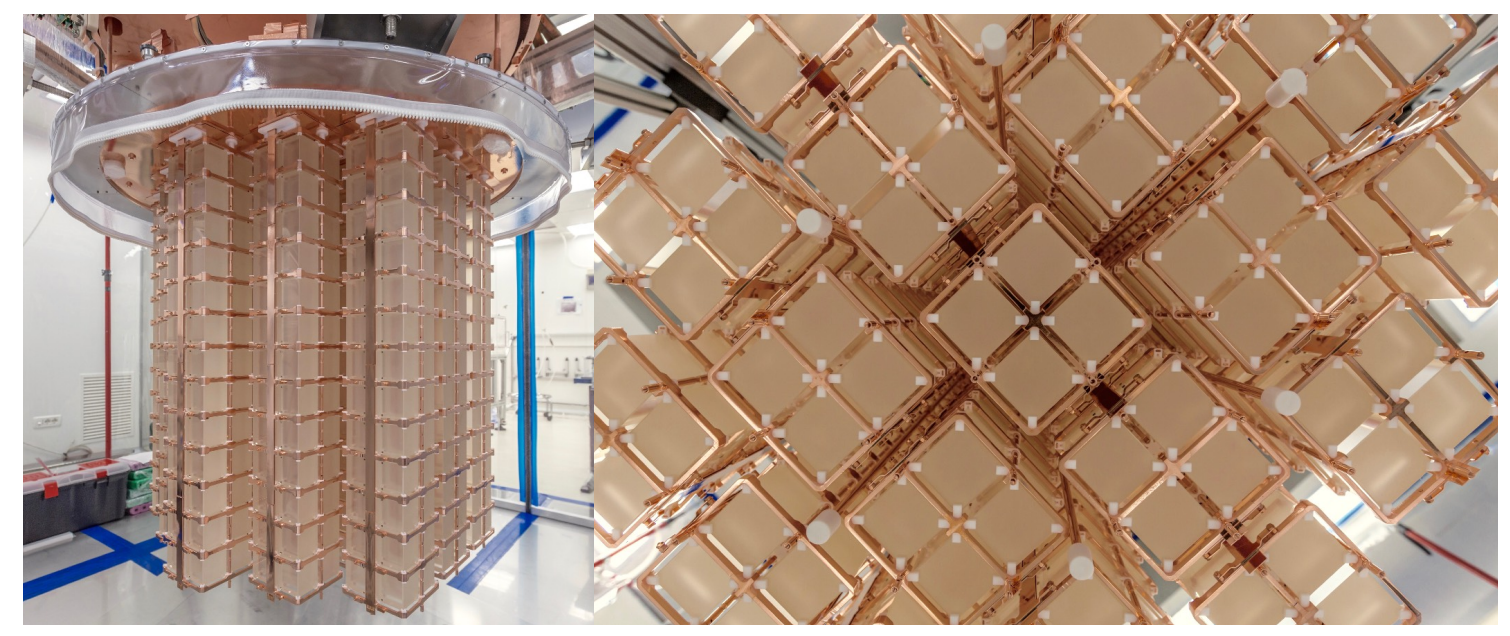

Figure 5: 988 bolometers configured in 19 towers attached on the top supporting plate of the CUORE cryostat (left). Bottom view of the towers, along with six capped guide tubes for the CUORE detector calibration (right).

\subsection{Beyond CUORE: CUPID}

As a next generation of CUORE, CUPID (CUORE Upgrade with Particle IDentification) is exploring various options towards lower background with particle discrimination with different isotopes, detectors, and sensors, as well as enriching Te. Vigorous R\&D efforts are pursued to this end [21]. CUPID aims to probe the entire Inverted hierarchy with $T_{1 / 2}^{0 v \beta \beta}$ sensitivity of $(2-5) \times$ $10^{27} \mathrm{y}$ in 10 years, which corresponds to $\mathrm{m}_{\beta \beta}$ sensitivity of 6-20 $\mathrm{meV}$ [22].

\section{References}

[1] B. Pontecorvo. Sov.Phys.JETP, 26:984-988, 1968.

[2] M.A. Luty. Phys.Rev., D45:455-465, 1992.

[3] D. R. Artusa et al. Eur. Phys. J. C, 74:2956, 2014.

[4] CUORE Official Website. http://cuore.lngs.infn.it/.

[5] F. Alessandria et al. Astropart. Phys., 35:839-849, 2012.

[6] C. Alduino et al. Phys. Rev. C, 93(4):045503, 2016.

[7] K. Alfonso et al. Phys. Rev. Lett., 115:102502, 2015. 
[8] C. Alduino et al. arXiv, 1609.01666, 2016.

[9] C. Alduino et al. JINST, 11(07):P07009, 2016.

[10] E. Andreotti et al. Astropart. Phys, 34:822, 2011.

[11] M. Agostini et al. Phys. Rev. Lett., 111(12):122503, 2013.

[12] A. Gando et al. Phys. Rev. Lett., 110(6):062502, 2013.

[13] J.B. Albert et al. Nature, 510:229, 2014.

[14] J. Kotila and F. Iachello. Phys. Rev. C, 85:034316, 2012.

[15] J. Menendez et al. Nucl. Phys. A, 818:139, 2009.

[16] F. Simkovic et al. Phys. Rev. C, 87:045501, 2013.

[17] J. Barea, J. Kotila, and F. Iachello. Phys. Rev. C, 91:034304, 2015.

[18] T. R. Rodriguez and G. Martinez-Pinedo. Phys. Rev. Lett, 105:252503, 2010.

[19] J. Hyvrinen and J. Suhonen. Phys. Rev. C, 91:024613, 2015.

[20] C. Savage, G. Gelmini, P. Gondolo, and K. Freese. JCAP, 0904:010, 2009.

[21] G. Wang et al. arXiv, 1504.03612, 2015.

[22] G. Wang et al. arXiv, 1504.03599, 2015. 